\title{
Containment Control for Sampled-data Multi-agent Systems Using the PD-like Protocols*
}

\author{
Lina Rong \\ School of Automation \\ Nanjing University of Posts \& Telecommunications \\ Nanjing, P. R. China \\ lnrong1128@163.com
}

\author{
Jinxing Lin \\ School of Automation \\ Nanjing University of Posts \& Telecommunications \\ Nanjing, P. R. China \\ jxlin2004@126.com
}

\begin{abstract}
This paper studies the distributed containment control problem in sampled-data multi-agent systems with directed network topologies. Different from the widely used containment controller which only adopts current information, we consider a distributed PD-like controller which can be regarded as an extension of the traditional PD-type controller for a single system. By augmenting the system dimension and applying the bilinear transformation, we obtain a necessary and sufficient condition of control gains which guarantees that the containment control problem can be solved. It is shown that under the proposed controller with appropriate control gains, the followers will eventually move into the convex hull spanned by the leaders. A numerical is provided to verify the theoretical results.
\end{abstract}

Keywords-multi-agent system; consensus; containment control; PD-like controller

\section{INTRODUCTION}

Over the last decade, the cooperative control of multi-agent systems has received remarkable attention, partly due to its wide applications such as formation control of robots, averaging in communication networks and distributed sensor networks [1]-[3]. A basic aspect to take into account in the cooperative control area is consensus, which means that a group of agents, starting from different initial states, agree on a certain value via local information sharing.

Building on the pioneering works [4] and [5], many researchers have been devoted to studying the consensus problem from different perspectives [4]-[8]. Roughly speaking, early literatures can be classified as leaderless consensus or leader-following consensus. The leader-following consensus problem is also mentioned as the distributed tracking, where a single stationary or dynamic leader is considered in an agent group. However, in some practical applications, there are several leaders and there is no information shared between them. In presence of multiple leaders, the distributed containment control problem arises, with the objective of designing containment controller which guarantees that the followers will eventually move into a given geometric space spanned by the leaders. Since the multiple leader concept was

This work was supported by the National Natural Science Foundation of China under grant 61304066, the China Postdoctoral Science Foundation under grant 2014M560438, the Postdoctoral Science Foundation of Jiangsu Province of China under grant 1402065B, the Natural Science Foundation of the Jiangsu Higher Education Institutions of China under grant 14KJB120008. introduced in [9], considerable works have been conducted on the containment control problem. The authors in [10] investigated the containment problem for first-order multiagent systems, providing necessary and sufficient conditions under which the containment control problem is solved. The second-order containment control was considered in [11], where distributed protocols were proposed for agent groups with both stationary and dynamic leaders, respectively. Efforts were made in [12] to further provide necessary and sufficient conditions for containment control of groups of first- and second-order agents. Among these works, however, a common feature is that only the current information is adopted in the containment controller design.

In this paper, we study the distributed containment control problem in sampled-data multi-agent systems with directed communication networks. Distinguished from the widely used protocol, we adopt a distributed PD-like protocol to solve the containment control problem. This protocol is partly motivated by the consensus protocol using outdated states in [8]. By augmenting the system dimension and applying the bilinear transformation, we provide a necessary and sufficient condition of control gains under which the containment control problem is solved. It is shown that the followers will eventually move into the convex hull spanned by the leaders with the proposed protocol. A numerical is provided to verify the theoretical results.

The rest of this paper is organized as follows. In Section II, the notation and preliminaries will be introduced. In Section III, the containment control problem using the PD-like protocol will be studied and the condition on the controller gains will be obtained. The illustrative example will be provided in Section $\mathrm{IV}$ and Section V will give a brief conclusion of this paper.

\section{NOTATION AND PRELIMINATIES}

We use a graph to describe the communication between a group of $N$ agents. We denote by $G=(E, V)$ a directed graph, where $V=\{1, \cdots, N\}$ is the nonempty set of nodes representing the $N$ agents, $E \subseteq V \times V$ is the edge set. An edge of $G$ is represented by a pair of distinct nodes $(i, j) \in E$, where node $i$ can obtain information from node $j$. Denote $N_{i}=\{j \in V \mid(i, j) \in E\}$ as the set of neighbors of node $i$. The adjacency matrix $A_{a d j}$ is defined such that $a_{i j}$ is the nonnegative weight of edge $(j, i)$. 
In this paper, we assume $a_{i j}=1$ if $(j, i) \in E$ and 0 otherwise and $a_{i i}=0$ for all $i \in\{1, \cdots N\}$. The in-degree of node $i$ is defined as $\operatorname{deg}_{i n}(i)=\sum_{j=1}^{N} a_{i j}$. The diagonal in-degree matrix is defined as $D=\operatorname{diag}\left\{\operatorname{deg}_{\text {in }}(\mathrm{i})\right\}$. It can be seen that the Laplacian matrix $L=\left[l_{i j}\right] \in \mathfrak{R}^{N \times N}$ can be represented as $L=D-A_{a d j}$.

Throughout this paper, we use $A \otimes B$ to represent the Kronecker product of the matrices $A$ and $B$. We write $\lambda_{i}(\cdot)$ to denote the $i$-th eigenvalue of a matrix.

We consider a group of $N$ agents governed by first-order sampled-data dynamics. Moreover, we assume that there are $M$ followers and $N-M$ leaders; denote the index set of the followers and the leaders as $F_{f}=\{1, \cdots, M\}$ and $R_{l}=\{M+1, \cdots, N\}$, respectively. In this paper, we need the following assumptions:

Assumption 2.1 Suppose that for each follower there exists at least one leader that has a directed path to that follower.

Assumption 2.2 Suppose that the sampling period $\varepsilon$ satisfies $0<\varepsilon<\frac{1}{\max _{i} \operatorname{deg}_{i n}(i)}$.

The agent dynamics is described as follows:

$$
x_{i}(k+1)=x_{i}(k)+\varepsilon u_{i}(k), \quad i \in F_{f} \cup R_{l}
$$

where $x_{i} \in \mathfrak{R}$ and $u_{i} \in \mathfrak{R}$ are the state and the input of agent $i$, respectively, and $\varepsilon$ is the sampling period of the agent.

The objective is to solve the distributed containment control problem, which can be described by the following definition.

Definition 2.1 ([13]). The distributed containment control problem is said to be solved for system (1) if there exists a controller using local information such that the states of the followers ultimately converge to the convex hull spanned by the states of the leaders for arbitrary initial conditions.

A widely used containment control protocol for multi-agent system (1) can be found in [10]. In this paper, we discuss the digital PD-like protocol. For the followers, the protocol can be provided as

$$
\begin{aligned}
u_{i}(k) & =\sum_{j \in N_{i}}\left[x_{i}(k)-x_{j}(k)\right] \\
& -\beta \sum_{j \in N_{i}}\left[\left(x_{i}(k)-x_{j}(k)\right)-\left(x_{i}(k-1)-x_{j}(k-1)\right)\right]
\end{aligned}
$$

where $i \in F_{f}, \beta \in \mathfrak{R}$ is the gain of the derivative part of the protocol. Notice that negative values are allowed in the gain $\beta$. And for the leaders, we set $u_{i}(k)=0$, where $i \in R_{l}$.

\section{MAIN RESULTS}

In this section, we focus on the containment control problem under the distributed PD-like protocols.
Lemma 3.1 ([14]). The complex coefficient polynomial $g(s)=s^{2}+a s+b$ is stable if and only if $\operatorname{Re}(a)>0$ and $\operatorname{Re}(s) \operatorname{Im}(a) \operatorname{Im}(b)+\operatorname{Re}^{2}(a) \operatorname{Re}(b)-\operatorname{Im}^{2}(b)>0$, where $a$ and $b$ are both complex numbers.

System (1) under protocol (2) can be rewritten as

$$
\begin{aligned}
x_{i}(k+1) & =x_{i}(k)-\varepsilon(1+\beta) \sum_{j \in N_{i}} l_{i j} x_{j}(k) \\
& =\varepsilon \beta \sum_{j \in N_{i}} l_{i j} x_{j}(k-1), \quad i \in F_{f} \\
x_{i}(k+1) & =x_{i}(k), \quad i \in R_{l}
\end{aligned}
$$

where $l_{i j}$ represents the $i$-th row and $j$-th column element of the Laplacian matrix $L$.

Let $\eta_{i}^{(1)}(k)=x_{i}(k), \eta_{i}^{(2)}(k)=x_{i}(k+1)$ and $\eta_{i}=\left[\eta_{i}^{(1)}, \eta_{i}^{(2)}\right]^{T}$. Then, multi-agent system (3)-(4) can be described as

$$
\begin{gathered}
\eta_{i}(k+1)=A \eta_{i}(k)+\sum_{j \in N_{i}} l_{i j} B \eta_{j}(k), \quad i \in F_{f} \\
\eta_{i}(k+1)=A \eta_{i}(k) \quad i \in R_{l} \\
\text { where } A=\left[\begin{array}{ll}
0 & 1 \\
0 & 1
\end{array}\right] \text { and } B=\left[\begin{array}{cc}
0 & 0 \\
\varepsilon \beta & -\varepsilon(1+\beta)
\end{array}\right] .
\end{gathered}
$$

Then, the collective network dynamics can be written as

$$
\eta(k+1)=\left(I_{N} \otimes A+L \otimes B\right) \eta(k)
$$

where $\eta=\left[\eta_{1}^{T}, \cdots, \eta_{N}^{T}\right]^{T}$ and

$$
L=\left[\begin{array}{cc}
L_{F F} & L_{F R} \\
0_{(N-M) \times M} & 0_{(N-M) \times(N-M)}
\end{array}\right] .
$$

Lemma 3.2. Let Assumption 2.1, 2.2 be satisfied. All the eigenvalues of $L_{F F}$ have positive real parts. Moreover, each entry of $-L_{F F}^{-1} L_{F R}$ is nonnegative with the sum of each row of $-L_{F F}^{-1} L_{F R}$ equal to one.

The proof is quite similar to Lemma 2 in [13] and thus is omitted here.

Let $F_{f}=\{1, \cdots, M\}=V_{1} \cup V_{2}$, where $V_{1}=\left\{i \mid \operatorname{Im}\left(\lambda_{i}(L)\right)=0, i \in F_{f}\right\}$ and $V_{2}=\left\{i \mid \operatorname{Im}\left(\lambda_{i}(L)\right) \neq 0, i \in F_{f}\right\}$. Now we provide the main results in this section.

Theorem 3.1. Let Assumption 2.1 and 2.2 be satisfied. The containment control problem of multi-agent system (1) under (2) can be solved when the following inequalities hold:

$$
-(2 \beta+1) \varepsilon\left|\lambda_{i}(L)\right|^{2}+2 \operatorname{Re}\left(\lambda_{i}(L)\right)>0, \quad i \in V_{1}
$$

and

$$
\beta+\frac{\operatorname{Re}\left(\lambda_{i}(L)\right)}{\varepsilon\left|\lambda_{i}(L)\right|^{2}}>0
$$




$$
2 \beta-1+\frac{2 \operatorname{Re}\left(\lambda_{i}(L)\right)}{\varepsilon\left|\lambda_{i}(L)\right|^{2}}>\left(2 \beta+1-\frac{2 \operatorname{Re}\left(\lambda_{i}(L)\right)}{\varepsilon\left|\lambda_{i}(L)\right|^{2}}\right) \Lambda_{i}
$$

where $i \in V_{2}$ and $\Lambda_{i}=\frac{\left(\beta \varepsilon\left|\lambda_{i}(L)\right|^{2}+\operatorname{Re}\left(\lambda_{i}(L)\right)\right)^{2}}{\operatorname{Im}\left(\lambda_{i}(L)\right)^{2}}$. Moreover, when the containment control problem is solved, the final states of the agents are as follows:

$$
\begin{gathered}
\lim _{k \rightarrow \infty} x_{F}(k)=-L_{F F}^{-1} L_{F R} x_{R}(0) \\
\lim _{k \rightarrow \infty} x_{R}(k)=x_{R}(0),
\end{gathered}
$$

where $x_{F}=\left[x_{1}, \cdots x_{M}\right]^{T}$ and $x_{R}=\left[x_{M+1}, \cdots x_{N}\right]^{T}$.

Proof. Notice that system (7) can be represented as

$$
\begin{aligned}
& \eta_{F}(k+1)=\left(I_{M} \otimes A+L_{F F} \otimes B\right) \eta_{F}(k)+L_{F R} \otimes \eta_{R}(k), \\
& \eta_{R}(k+1)=\left(I_{M} \otimes A\right) \eta_{R}(k)
\end{aligned}
$$

For the leaders, it can be seen from (6) that at time $k$, the state $\eta_{i}(k)$ is obtained by

$$
\eta_{i}(k)=A^{k} \eta_{i}(0) .
$$

Notice that $A^{k}=A$, it can be derived that $\eta_{i}(k)=A \eta_{i}(0)$, which further implies that $\lim _{k \rightarrow \infty} x_{R}(k)=x_{R}(0)$.

For the followers, let $\xi(k)=\eta_{F}(k)+\left(L_{F F}^{-1} L_{F R} \otimes I_{2}\right) \eta_{R}(k)$. It is seen that $\eta_{F}(k)=-\left(L_{F F}^{-1} L_{F R} \otimes I_{2}\right) \eta_{R}(k)$ if and only if $\xi(k)=0$. In virtue of Lemma 3.2, we know that the containment control problem can be solved if and only if $\lim _{k \rightarrow \infty} \xi(k)=0$. Then we have

$$
\begin{gathered}
\xi(k+1)=\left(I_{M} \otimes A+L_{F F} \otimes B\right) \eta_{F}(k)+\left(L_{F R} \otimes B\right) \eta_{R}(k) \\
+\left(L_{F F}^{-1} L_{F R} \otimes I_{2}\right)\left(I_{N-M} \otimes A\right) \eta_{R}(k)
\end{gathered}
$$

By noticing that $L_{F R} \otimes B=\left(L_{F F} \otimes B\right)\left(L_{F F}^{-1} L_{F R} \otimes I_{2}\right)$ and $\left(L_{F F}^{-1} L_{F R} \otimes I_{2}\right)\left(I_{N-M} \otimes A\right)=\left(I_{M} \otimes A\right)\left(L_{F F}^{-1} L_{F R} \otimes I_{2}\right)$,

we can get that

$$
\xi(k+1)=\left(I_{M} \otimes A+L_{F F} \otimes B\right) \xi(k) .
$$

As a result, we have that

$$
\xi(k+1)=\left(I_{M} \otimes A+L_{F F} \otimes B\right)^{k} \xi(0) .
$$

Thus, system (17) is asymptotically stable if and only if $\Xi:=I_{M} \otimes A+L_{F F} \otimes B$ is Schur stable.

Next, we focus on the matrix $\Xi$. The eigenvalues of $\Xi$ satisfy that

$$
\begin{aligned}
& \operatorname{det}\left[\lambda I_{2 M}-\Xi\right] \\
= & \prod_{i=1}^{M} \operatorname{det}\left[\lambda I_{2}-\left(A+\lambda_{i}(L) B\right)\right] \\
= & \prod_{i=1}^{M}\left\{\lambda^{2}+\left[\lambda_{i}(L) \varepsilon(1+\beta)-1\right] \lambda-\lambda_{i}(L) \varepsilon \beta\right\}=0
\end{aligned}
$$

which equals to that

$$
\lambda^{2}+\left[\lambda_{i}(L) \varepsilon(1+\beta)-1\right] \lambda-\lambda_{i}(L) \varepsilon \beta=0,
$$

where $i=1, \cdots, M$. Applying bilinear transformation $\lambda=\frac{\omega+1}{\omega-1}$, we have that

$$
\lambda_{i}(L) \varepsilon \omega^{2}+21\left(+\lambda_{i}(L) \varepsilon \beta\right) \omega+\left(2-\lambda_{i}(L) \varepsilon-2 \lambda_{i}(L) \varepsilon \beta\right)=0
$$

Thus, we can conclude that system (17) is asymptotically stable if and only if the solutions of (20) have negative real parts.

As $\varepsilon>0$ and $\operatorname{Re}\left(\lambda_{i}(L)\right)>0, i=1, \cdots M$, equation (20) is equivalent to that

$$
\omega^{2}+\rho_{i} \omega+\gamma_{i}=0
$$

where

$$
\begin{gathered}
\rho_{i}=2 \beta+\frac{2\left(\operatorname{Re}\left(\lambda_{i}(L)\right)-j \operatorname{Im}\left(\lambda_{i}(L)\right)\right)}{\varepsilon\left|\lambda_{i}(L)\right|^{2}}, \\
\gamma_{i}=-2 \beta-1+\frac{2\left(\operatorname{Re}\left(\lambda_{i}(L)\right)-j \operatorname{Im}\left(\lambda_{i}(L)\right)\right)}{\varepsilon\left|\lambda_{i}(L)\right|^{2}} .
\end{gathered}
$$

From (22)-(23), it is seen that

$$
\begin{aligned}
& \operatorname{Re}\left(\rho_{i}\right)=\frac{2 \beta \varepsilon\left|\lambda_{i}(L)\right|^{2}+2 \operatorname{Re}\left(\lambda_{i}(L)\right)}{\varepsilon\left|\lambda_{i}(L)\right|^{2}}, \\
& \operatorname{Re}\left(\gamma_{i}\right)=\frac{-(2 \beta+1) \varepsilon\left|\lambda_{i}(L)\right|^{2}+2 \operatorname{Re}\left(\lambda_{i}(L)\right)}{\varepsilon\left|\lambda_{i}(L)\right|^{2}}, \\
& \operatorname{Im}\left(\rho_{i}\right)=\operatorname{Im}\left(\gamma_{i}\right)=\frac{-2 \operatorname{Im}\left(\lambda_{i}(L)\right)}{\varepsilon\left|\lambda_{i}(L)\right|^{2}} .
\end{aligned}
$$

(26)

Notice that $\operatorname{Im}\left(\rho_{i}\right)=\operatorname{Im}\left(\gamma_{i}\right)$. Moreover, according to (26), we have that $\operatorname{Im}\left(\lambda_{i}(L)\right)=0$ if and only if $\operatorname{Im}\left(\rho_{i}\right)=\operatorname{Im}\left(\gamma_{i}\right)=0$.

When $i \in V_{1}$, using Lemma 3.1, the roots of (21) have negative real parts if only if $\operatorname{Re}\left(\gamma_{i}\right)>0$, i.e.,

$$
-(2 \beta+1) \varepsilon\left|\lambda_{i}(L)\right|^{2}+2 \operatorname{Re}\left(\lambda_{i}(L)\right)>0
$$

When $i \in V_{2}$, using Lemma 3.1, we have that the roots of (21) have negative real parts if and only if

$$
\begin{aligned}
& \operatorname{Re}\left(\rho_{i}\right)>0, \\
& \operatorname{Re}\left(\rho_{i}\right)+\operatorname{Re}\left(\gamma_{i}\right) \frac{\operatorname{Re}\left(\rho_{i}\right)^{2}}{\operatorname{Im}\left(\rho_{i}\right)^{2}}>1
\end{aligned}
$$

As a result, for $i \in V_{2}$, the roots of (21) have negative real parts if and only if the parameters satisfy (8)-(10).

Thus, we can conclude that system (17) is asymptotically stable if and only if the parameters satisfy (8)-(10), which 
further implies that $\lim _{k \rightarrow \infty} x_{F}(k)=-L_{F F}^{-1} L_{F R} x_{R}(0)$ if and only if the parameters satisfy (8)-(10).

The proof can be completed.

\section{NUMERICAL EXAMPLES}

Example 4.1. Consider a network of 6 agents. The interaction topology of the agents is shown in Fig.1. The corresponding Laplacian matrix can be written as

$$
L=\left[\begin{array}{cccccc}
2 & -1 & 0 & -1 & 0 & 0 \\
0 & 2 & 0 & -1 & -1 & 0 \\
-1 & 2 & -1 & 0 & 0 & 0 \\
0 & -1 & -1 & 3 & 0 & -1 \\
0 & 0 & 0 & 0 & 0 & 0 \\
0 & 0 & 0 & 0 & 0 & 0
\end{array}\right] .
$$

Let $\varepsilon=0.2$. It is seen that Assumption 2.1 and 2.2 are satisfied. The initial condition is given as $\mathrm{x}(0)=$ $[-1,10,-2,5,7,1]^{T}$. When $\beta=0.5$, it is seen that the parameters satisfy (8)-(10). The state trajectories of agents are shown in Fig.2.

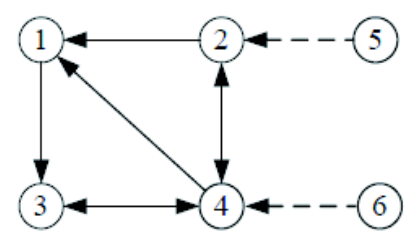

Fig. 1. The communication tolology of a group of 6 agents.

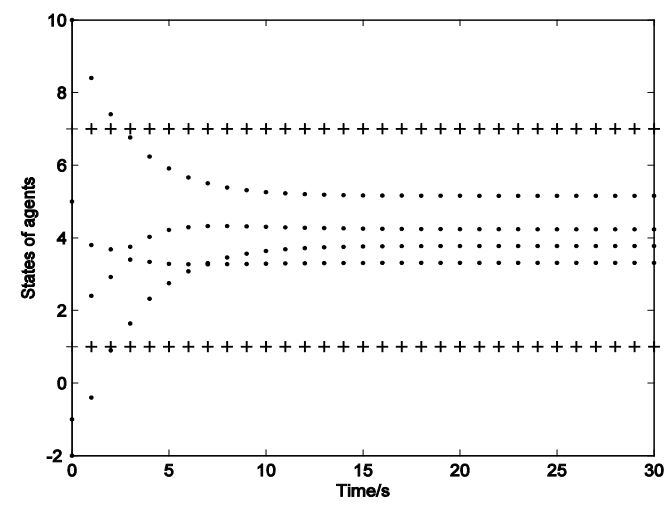

Fig. 2. The state trajectories with $\beta=0.5$.

\section{CONCLUSION}

We have studied the distributed containment control problem in sampled-data multi-agent systems with directed network topologies. Distinguished from the widely used containment controller which only exploits current information, we have further considered a distributed PD-like controller. By augmenting the system dimension and applying the bilinear transformation, we have obtained a necessary and sufficient condition of control gains which guarantees that the containment control problem can be solved. A numerical have been provided to verify the theoretical results.

\section{REFERENCES}

[1] Z. Lin, M. Broucke, and B. Francis, "Local control strategies for groups of mobile autonomous agents," IEEE Trans. Autom. Control, vol. 49, no. 4, pp. 622-629, 2004.

[2] L. Xiao and S. Boyd, "Fast linear iterations for distributed averaging," System \& Control Letters, vol. 53, no. 1, pp. 65-78, 2004.

[3] R. Olfati-Saber and J.S. Shamma, "Consensus filters for sensor networks and distributed sensor fusion," 44th IEEE Conference on Decision and Control, 2005, pp. 6698-6703.

[4] R. Olfati-Saber and R. M. Murray, "Consensus problems in networks of agents with switching topology and time-delays," IEEE Trans. Autom. Control, vol. 49, no. 9, pp. 1520-1533, 2004.

[5] W. Ren and R. W. Beard, "Consensus seeking in multiagent systems under dynamically changing interaction topologies," IEEE Trans. Autom. Control, vol. 50, no. 5, pp. 655-661, 2005.

[6] R. Olfati-Saber, J.A. Fax, and R. M. Murray, "Consensus and Cooperation in networked multi-agent systems,". Proceedings of the IEEE, vol. 95, no. 1, pp. 215-233, 2007.

[7] Z. Li, Z. Duan, G. Chen, and L. Huang, "Consensus of multiagent systems and synchronizaiton of complex networks: A unified viewpoint," IEEE Trans. Circuits Syst. I, Regul. Pap., vol. 57, no. 1, pp. 213-224, 2010.

[8] J. Li, S. Xu, Y. Chu, and H. Wang, "Distributed average consensus control in networks of agents using outdated states," IET Control Theory Appl., vol. 4, no.5, pp. 746-758, 2010.

[9] M. Ji, G. Ferrari-Trecate, M. Egerstedt, and A. Buffa, "Containment control in mobile networks," IEEE Trans. Autom. Control, vol. 53, no. 8, pp. 1299-1305, 2008.

[10] Y. Cao and W. Ren, "Containment control with multiple stationary or dynamic leaders under a directed interaction graph," Proceedings of the 48th IEEE Conference on Decision and Control and the 28th Chinese Control Conference, Shanghai, China, 2009, pp. 3014-3019.

[11] Y. Cao, D. Stuart, W. Ren, and Z. Meng, "Distributed containment control for double-integrator dynamics: algorithms and experiments," Proceedings of the 2010 American Control Conference, 2010, pp. 38303835.

[12] H. Liu, G. Xie, and L. Wang, "Necessary and sufficient conditions for containment control of networked multi-agent systems," Automatica, vol. 48, pp. 1415-1422, 2012.

[13] Z. Li, W. Ren, X. Liu, and M. Fu, "Distributed containment control of multi-agent systems with general linear dynamincs in the presence of multiple leaders," Int. J. Robust Nonlinear Control, vol. 23, no. 5, pp. 534-547, 2013.

[14] P. C. Parks and V. Hahn, Stability theory, Prentice Hall, 1990 